
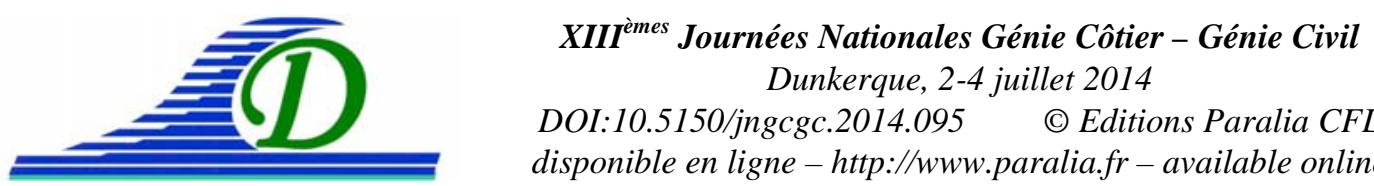

DOI:10.5150/jngcgc.2014.095 @ Editions Paralia CFL

disponible en ligne - http://www.paralia.fr - available online

\title{
Contribution du setup induit par les vagues dans la surcote associée à la tempête Klaus
}

\author{
Gael ARNAUD ${ }^{1}$, Xavier BERTIN ${ }^{1}$
}

\author{
1. UMR 7266 LIENSs, CNRS-Université de La Rochelle, \\ 2 rue Olympe de Gouge, 17000 La Rochelle, France. \\ gael.arnaud@univ-Ir.fr ; xavier.bertin@univ-Ir.fr
}

\section{Résumé :}

La modélisation des surcotes de tempête et des submersions marines est un enjeu considérable pour la protection des biens et des personnes compte tenu du danger qu'elles représentent. Même si les mécanismes principaux sont bien connus, la contribution liée aux vagues n'est pas systématiquement prise en compte.

Dans cette étude, nous réalisons une simulation rétrospective de la tempête Klaus qui a générée une surcote de $1,7 \mathrm{~m}$ dans le bassin d'Arcachon et de $1 \mathrm{~m}$ dans le port de Bayonne.

Afin d'analyser les processus physiques expliquant ces observations, nous avons implémenté un système de modélisation numérique de surcote, réalisant le couplage entre un code de circulation hydrodynamique (SELFE) et un modèle spectral d'état de mer (WWMII). La comparaison entre les mesures disponibles et nos résultats numériques montre que le modèle est capable de reproduire les paramètres de vagues avec des erreurs normalisées de l'ordre de 10 à $15 \%$ et les surcotes avec des erreurs variant de 0,1 à $0,15 \mathrm{~m}$. L'analyse des résultats numériques nous permet alors de quantifier la contribution du setup lié au vagues dans la surcote et la capacité de celui-ci à se propager en dehors des zones de déferlement, comme c'est le cas dans le bassin d'Arcachon ou dans le port de Bayonne.

Mots-clés : Surcote, Klaus, Tempête, Vagues, Tensions de radiation, Modélisation numérique.

\section{Introduction}

Les submersions marines sont l'une des catastrophes naturelles générant les plus importantes pertes humaines et dégâts matériels. L'augmentation de la pression démographique sur les littoraux conjointement à l'élévation du niveau de la mer risque d'aggraver cette situation. Les récentes submersions marines associées aux tempêtes Katrina (2005) et Ike (2008) dans le Golfe du Mexique, Sandy (2012) dans l'état de New York (KANTHA, 2013) ou le typhon Haiyan aux Philippines illustrent l'importance de ce phénomène. Les submersions marines sont associées à des niveaux marins extrêmes, résultant en général de la superposition d'une forte surcote et de marées hautes de fortes amplitudes. À titre d'exemple, la surcote qui a dévasté la 


\section{Thème 7 - Risques côtiers}

Nouvelle Orleans pendant l'ouragan Katrina a atteint une cote record de plus de 8,5 m (IRISH \& RESIO, 2010). Ces événements soulignent la nécessité de mieux comprendre la génération des surcotes afin d'améliorer leur prédiction. Les mécanismes principaux qui contribuent à la génération d'une surcote de tempête sont établis depuis plusieurs décennies et sont essentiellement liés à la contrainte de surface exercée par le vent et à la pression atmosphérique (baromètre inverse). En milieu peu profond l'effet du vent devient en général prédominant sur les effets de la pression atmosphérique et de ce fait, la présence d'un plateau continental large est un facteur aggravant.

Plus récemment, des études ont mis en évidence l'importance de la contribution des vagues dans les surcotes de tempête. Ainsi, pour des conditions de vent données, l'état de mer peut augmenter la contrainte de frottement dans le cas d'un état de mer jeune favorisant ainsi le transfert de quantité de mouvement de l'atmosphère à l'océan et contribuant à augmenter la surcote (NICOLLE, 2006 ; NICOLLE et al., 2009 ; BERTIN et al., 2012).

Plus près des côtes, le setup induit par les vagues peut également contribuer à la surcote. Dans les zones de déferlement, les gradients des tensions de radiation génèrent des courants et une surélévation du plan d'eau jusqu'à ce qu'un équilibre se produise avec le gradient de pression barotrope (LONGUET-HIGGINS \& STEWART, 1964). Cette surélévation est sensible sur les plages et des études ont montré qu'elle pouvait également se propager dans les lagunes (DODET et al., 2013; TORRESFREYERMUTH et al., 2012). Cependant, la prise en compte du setup dans les modèles régionaux de surcote requiert une résolution spatiale métrique à déca-métrique, ce qui constitue un défi en termes de temps de calcul et explique probablement le peu d'études publiées à ce sujet.

Pour cette étude, nous proposons de réaliser une simulation rétrospective de la surcote et de l'état de mer induits par la tempête Klaus dans la partie Sud du Golfe de Gascogne. Cette tempête présente un intérêt particulier du fait de conditions de vagues exceptionnelles et d'un vent très fort. L'utilisation d'un système de modélisation entièrement couplé vagues/courants, nous permet d'étudier et de quantifier la contribution du setup des vagues dans la surcote générée lors de la tempête dans le Sud du Golfe de Gascogne.

\section{Cas d'étude}

\subsection{Description de la zone d'étude}

Notre étude s’intéresse au quart Sud-Est du Golfe de Gascogne. La zone ainsi modélisée va de la pointe du Médoc en France à Santander en Espagne et se focalise sur le bassin d'Arcachon et Bayonne (Figure 1). Le plateau continental de cette zone se rétrécit du Nord au Sud de $70 \mathrm{~km}$ au large du bassin d'Arcachon à une vingtaine de kilomètres au large de Bayonne. Le régime de marée est méso-tidal semi-diurne. Les analyses du 


\section{XIII ${ }^{\text {èmes }}$ Journées Nationales Génie Côtier - Génie Civil \\ Dunkerque, 2-4 juillet 2014}

régime d'agitation de la zone montrent que les hauteurs significatives des vagues $H_{s}$ annuelles moyennes varient entre 1,36 m au Nord au large de Biscarrosse et $1,57 \mathrm{~m}$ au Sud au large de Bayonne. Les périodes pic se distribuent respectivement entre 11,55 s et 9,59 s et les directions au pic sont majoritairement de $301,21^{\circ}$ au Nord et $301,9^{\circ}$ au Sud (ABADIE et al., 2005).

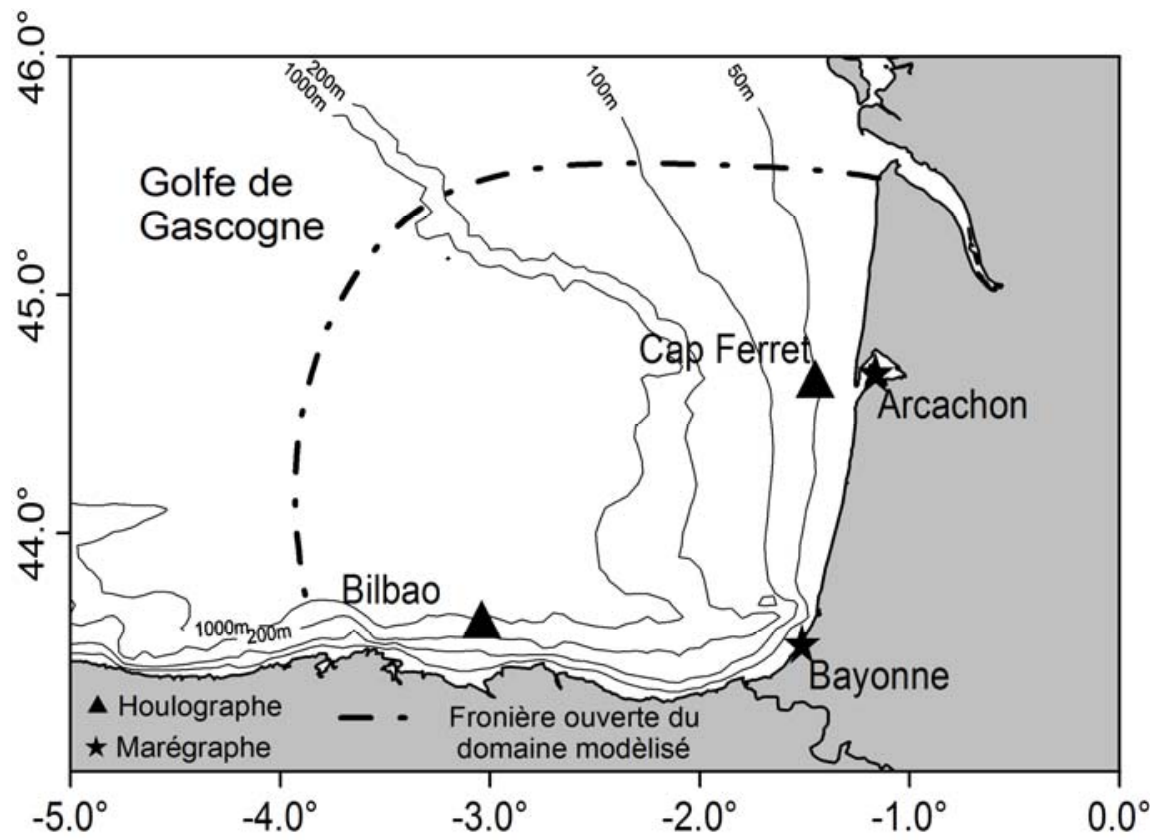

Figure 1. Localisation de la zone d'étude et des points de mesures.

\subsection{La tempête Klaus}

La tempête Klaus a frappé les côtes françaises dans la nuit du 23 au 24 janvier 2009. La trajectoire de la tempête a suivi une direction ONO-ESE. Le centre de la dépression est passé au-dessus de La Rochelle alors que les vents les plus forts ont été mesurés à Arcachon. Avec une pression minimale enregistrée de 965,5 hPa lors de son passage à La Rochelle, cette tempête a été exceptionnelle par la vitesse des vents qui ont atteint $36 \mathrm{~m} \mathrm{~s}^{-1}$ au Cap Ferret, pour une pression de $976 \mathrm{hPa}$. Plus au Sud, les vents étaient plus modérés et l'aéroport de Biarritz a enregistré une vitesse maximale de $21 \mathrm{~m} \mathrm{~s}^{-1}$ de direction $270^{\circ}$ avec une pression minimale de $983,6 \mathrm{hPa}$. La taille des vagues dans le Golfe de Gascogne a été aussi exceptionnelle avec des $H_{s}$ supérieures à $13 \mathrm{~m}$ enregistrées au houlographe de Bilbao avec une période pic de $16 \mathrm{~s}$ pour une direction de $293^{\circ}$. Le houlographe du Cap Ferret n'a malheureusement pas enregistré au plus fort de la tempête.

\section{Description du modèle}

Le système de modélisation que nous utilisons réalise le couplage complet entre le modèle de circulation hydrodynamique SELFE (ZHANG \& BAPTISTA, 2008) et le 


\section{Thème 7 - Risques côtiers}

modèle spectral de vagues WWMII (ROLAND et al., 2009). Les deux modèles partagent les mêmes grilles non-structurées et décomposition de domaine. Le maillage de la zone a une résolution qui varie de $7 \mathrm{~km}$ à la frontière ouverte à $50 \mathrm{~m}$ au niveau des zones d'intérêt (Arcachon et Bayonne). La méthode Eulérienne-Lagrangienne (ELM) de résolution de l'advection (ZHANG \& BAPTISTA, 2008) permet de s'affranchir des conditions CFL et le pas de temps de calcul est fixé à $60 \mathrm{~s}$ pour les deux modèles. La durée de la simulation est de 7 jours et démarre le 19 janvier 2009, 5 jours avant le pic de la tempête Klaus.

\subsection{Modèle de vague}

Le modèle de vague Wind Wave Model II (WWMII) est un modèle spectral qui résout l'équation de conservation de la densité d'action des vagues. Dans les simulations réalisées ici, les termes sources pour la génération des vagues et la dissipation par moutonnement sont implémentés selon la formulation WAM Cycle 4 (BIDLOT et al., 2007). Le frottement au fond utilise une paramétrisation JONSWAP et le déferlement bathymétrique la formulation de BATTJES \& JANSSEN (1978).

Le déferlement bathymétrique est paramétré par l'indice de déferlement $\gamma=H_{m} / h_{b}$ où $H_{m}$ et $h_{b}$ représente respectivement la hauteur maximale des vagues et la profondeur au point de déferlement. La valeur par défaut du modèle est $\gamma=0,73$ et donne des valeurs de surcote beaucoup trop importantes au moment du pic de la tempête. Plusieurs travaux ont montré que des valeurs de $\gamma$ plus faibles étaient plus adaptées à des houles plus cambrées et APOTSOS et al. (2008) montrent que ces valeurs peuvent s'étaler entre 0,4 et 0,7 . Compte tenu des conditions particulièrement exceptionnelles de la tempête ( $H_{s}=13 \mathrm{~m}$ avec $16 \mathrm{~s}$ de période), la valeur de $\gamma$ a été ajustée à 0,4.

Le long de la frontière ouverte, WWMII est forcé par des séries temporelles de spectres directionnels, interpolés linéairement depuis un modèle régional de vague reposant sur le code Wave Watch III et décrit dans DODET et al. (2010). Le modèle est aussi forcé sur l'ensemble du domaine par les champs de vents issus de la réanalyse CFSR (SAHA et al., 2010) avec une résolution spatiale de $38 \mathrm{~km}$ et une résolution temporelle horaire. Enfin, les tensions de radiations sont calculées selon la formulation de BATTJES (1978) et leur gradient est calculé selon une méthode d'éléments finis utilisant une fonction de forme spline cubique.

\subsection{Modèle de circulation hydrodynamique}

Le modèle de circulation hydrodynamique utilisé dans cette étude est le code SELFE en en mode 2DH barotrope (ZHANG et al., 2011).

Les termes sources pris en compte sont les gradients de la pression atmosphérique, la contrainte de surface, qui est calculée selon la formulation de POND \& PICKARD (1998) et le gradient des tensions de radiation fourni par le modèle de vague. Le long de sa frontière ouverte, SELFE est forcé par les amplitudes et phases des 16 principaux 


\section{XIII ${ }^{\text {èmes }}$ Journées Nationales Génie Côtier - Génie Civil \\ Dunkerque, 2-4 juillet 2014}

constituants de la marée astronomique, interpolés linéairement depuis le modèle régional de marée de BERTIN et al. (2012). Un baromètre inverse est également appliqué le long de la frontière ouverte afin de prendre en compte la surcote déjà présente en dehors du domaine modélisé.

\section{Résultats du modèle}

\subsection{Vagues}

Pour valider les résultats du modèle les paramètres de vagues utilisés sont issus des houlographes de Bilbao et Cap Ferret (figures 2 et 3) donnant respectivement les $H_{s}$, la période pic et la direction de pic toutes les heures et la hauteur $H_{1 / 3}$, la période associée $T_{H 1 / 3}$ et la direction au pic toutes les demi-heures. L'enregistrement de Bilbao s'arrête au plus fort de la tempête et celui d'Arcachon n'est pas disponible pendant la tempête. Les erreurs mentionnées sur les graphiques sont normalisées par la moyenne des données et sont de l'ordre de $10 \%$ sur $H_{s}$ et sur la période à Bilbao de $14 \%$ sur $H_{1 / 3}$ et $10 \%$ sur la période au Cap Ferret.

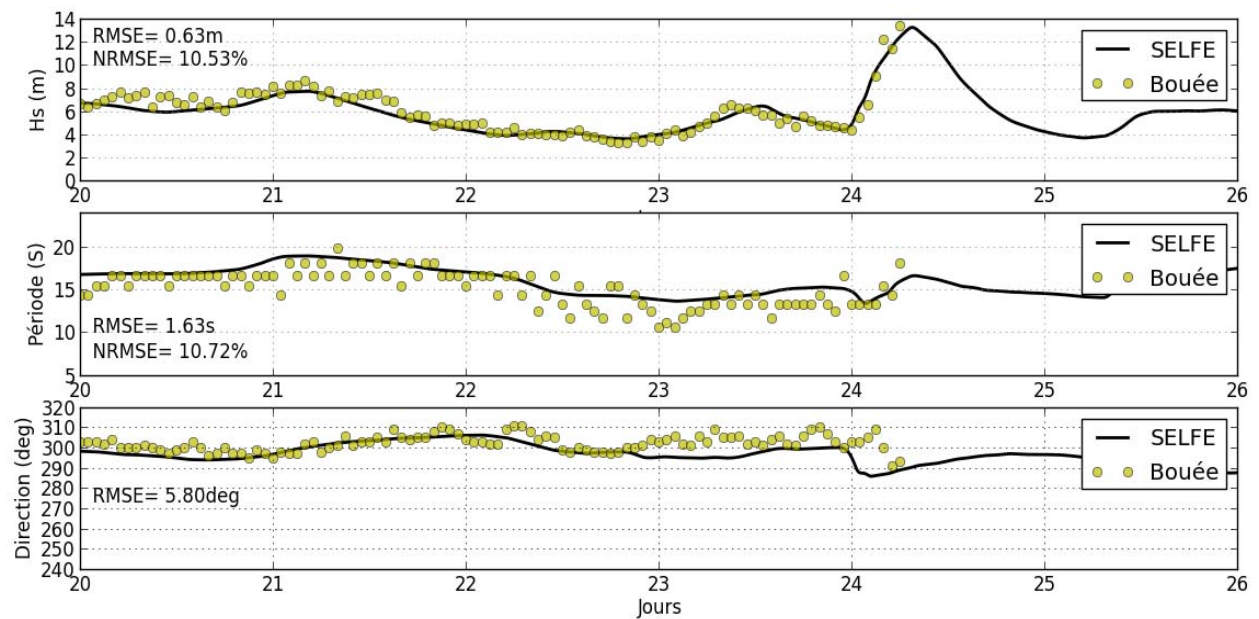

Figure 2. Comparaison entre les Hs, Tp et Dir mesurées (vert) et modélisées (noir) à Bilbao. 


\section{Thème 7 - Risques côtiers}
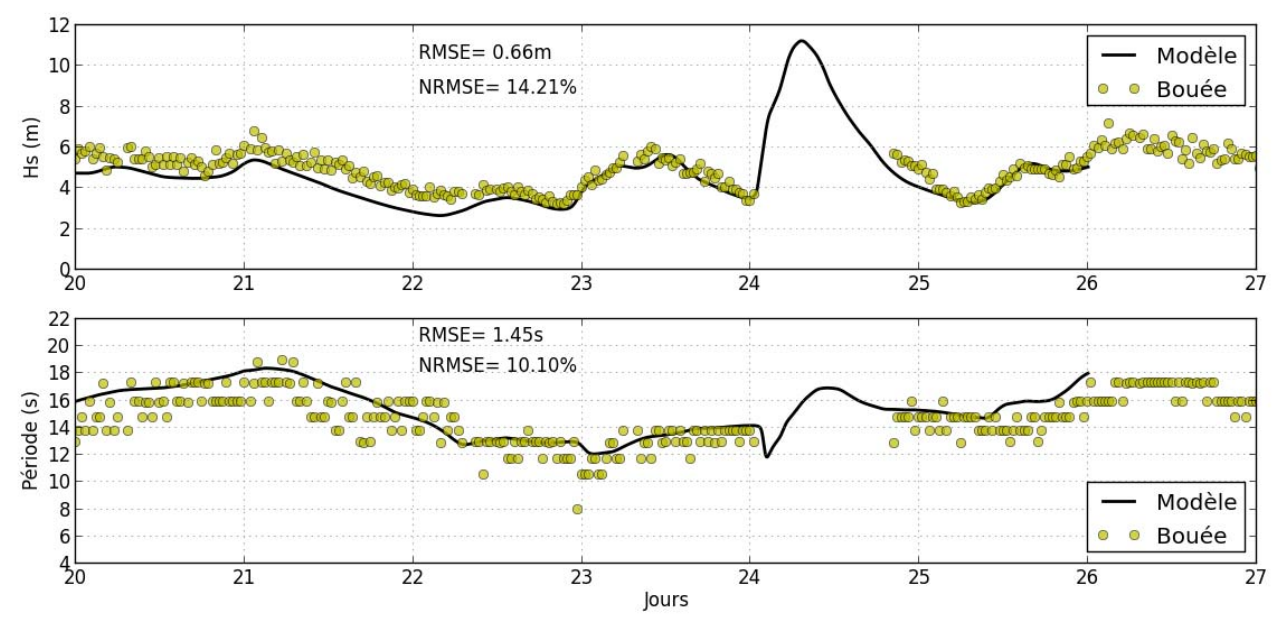

Figure 3. Comparaison entre les $H_{1 / 3}, T_{1 / 3}$ mesurées (vert) et modélisées (noir) au Cap Ferret.

\subsection{Surcote}

Les hauteurs d'eau aux points de validation sont issues des enregistrements des marégraphes d'Arcachon et de Bayonne mis à disposition sur le portail REFMAR (http://refmar.shom.fr). Les constituants astronomiques sont extraits par analyse harmonique exécutée par le code TAPPy sur des enregistrements d'une année. La marée astronomique est ensuite recomposée à partir de ces constituants, après avoir éliminé le constituant Sa, dont l'origine est essentiellement atmosphérique et thermos-stérique. La surcote est finalement calculée par soustraction de ce signal à l'enregistrement des marégraphes. Les surcotes du modèle sont calculées en soustrayant les résultats d'une simulation avec la marée seule aux résultats des simulations où sont pris en compte les forçages (vents seuls et vents, marées, vagues et interaction vagues/courants).

Les surcotes calculées dans le modèle avec et sans l'effet des vagues sont comparées à la surcote déduite des enregistrements des marégraphes et présentées pour les deux points de mesure sur la figure 4. Les surcotes simulées avec l'effet des vagues reproduisent bien mieux les surcotes observées, avec une erreur quadratique moyenne diminuée de moitié. Sans l'effet des vagues, la surcote est systématiquement sous-estimée aux deux stations et il manque $40 \mathrm{~cm}$ au plus fort de la tempête à Arcachon et à jusqu'à $60 \mathrm{~cm}$ à Bayonne. 


\section{XIII ${ }^{\text {èmes }}$ Journées Nationales Génie Côtier - Génie Civil \\ Dunkerque, 2-4 juillet 2014}
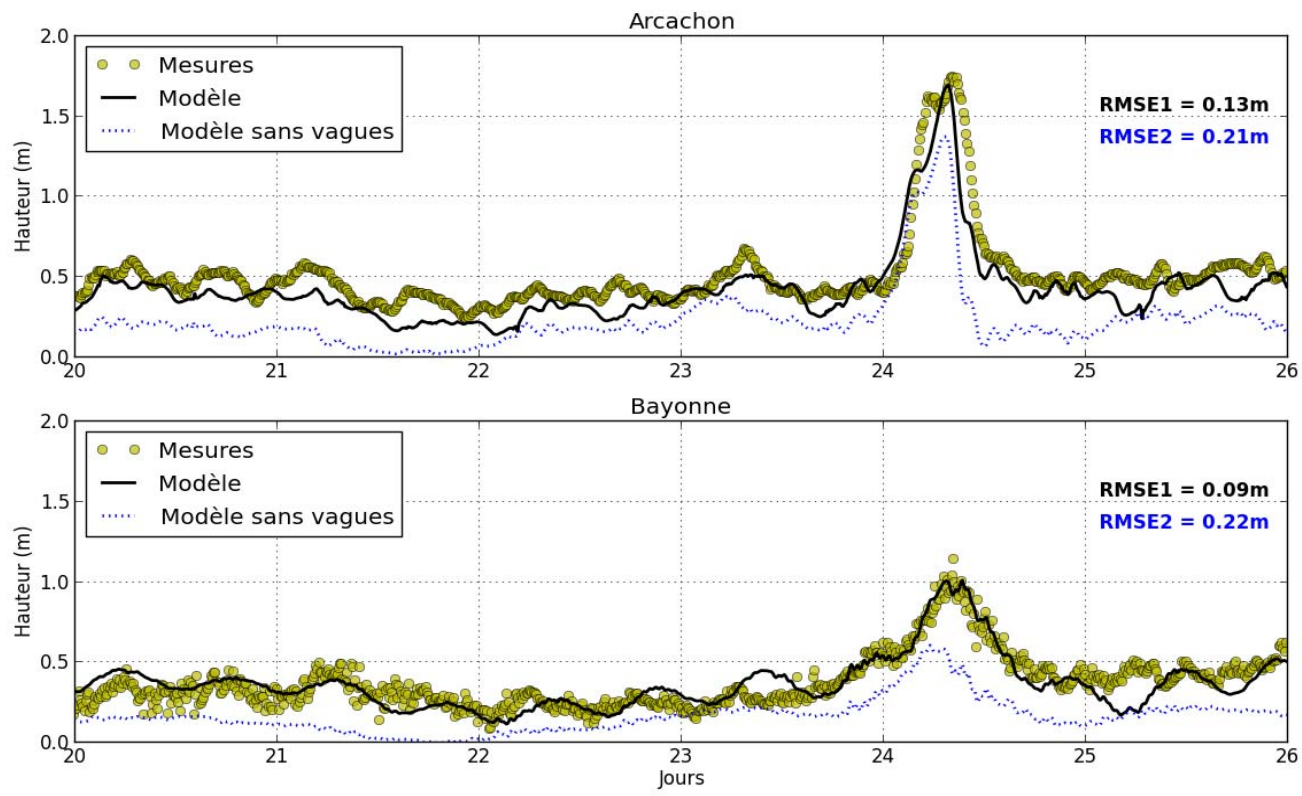

Figure 4. Comparaison entre les surcotes obtenues lors des simulations avec (noir) et sans (bleu) le setup des vagues et les surcotes observées (vert). Les erreurs mentionnées correspondent aux erreurs entre les mesures et le modèle avec setup (RMSE1) et sans setup des vagues (RMSE2).

\section{Discussion et conclusions}

Les paramètres synthétiques d'état de mer pendant l'évènement de tempête aux stations Cap Ferret et Bilbao sont bien représentés à la fois en amplitude et temporellement, indiquant une bonne reproduction des conditions de tempête dans la zone modélisée.

Les simulations présentées montrent une bonne reproduction des surcotes de tempête enregistrées à Arcachon et Bayonne avec des erreurs de l'ordre de $10 \mathrm{~cm}$ sur l'ensemble de la simulation. Bien qu'il s'agisse d'une surcote exceptionnelle, les pics de surcote pendant l'évènement de tempête sont également bien représentés avec moins de $5 \mathrm{~cm}$ d'erreur. La contribution du setup des vagues dans les surcote des deux sites est globalement identique (jusqu'à $40 \mathrm{~cm}$ ), ce qui s'explique assez bien par les zones de déferlement importantes qui se développent à l'embouchure des estuaires dans lesquels se trouvent ces deux stations : au niveau du delta de jusant à l'embouchure du bassin d'Arcachon et au niveau des plages d'Anglet à l'embouchure de l'Adour. Dans les deux cas, le setup issu du déferlement dans ces zones se propage hors de la zone de déferlement et contribue à l'élévation du niveau d'eau à l'intérieur de la lagune d'Arcachon et dans l'Adour.

La surcote qui n'est pas liée à l'effet des vagues (figure 4 modèle sans vagues), est nettement plus importante à Arcachon qu'à Bayonne. Ceci peut s'expliquer par la vitesse du vent qui était bien moins importante à Bayonne, mais aussi par la largeur du plateau 


\section{Thème 7 - Risques côtiers}

continental plus importante à la latitude d'Arcachon. Les effets liés au vent deviennent alors plus importants et la surcote d'origine atmosphérique atteint $1,3 \mathrm{~m}$ à Arcachon alors qu'elle n'est que de $60 \mathrm{~cm}$ à Bayonne, les effets de la pression atmosphérique contribuant à hauteur de 0,37 m à Arcachon et 0,30 m à Bayonne.

La paramétrisation du critère de déferlement dans le modèle de vague joue un rôle important dans l'obtention de ces résultats. Avec une valeur de $\gamma=0,73$ (valeur par défaut du modèle), la surcote atteint $2 \mathrm{~m}$ à la station d'Arcachon et devient trop importante par rapport aux observations. Cependant, une calibration rigoureuse de ce paramètre impliquerait de réaliser des mesures dans la zone de déferlement, ce qui représente un défi technique pour des conditions d'agitations exceptionnelles comme celles de la tempête Klaus. Les faibles valeurs de $\gamma$ suggérées par notre étude pour des vagues de hauteurs exceptionnelles devront être vérifiées sur d'autres sites.

Cette étude a montré que le setup des vagues pouvait participer à la surcote totale à hauteur de 50\% pendant une tempête, même en dehors des zones de déferlement. Ce processus doit donc impérativement être mieux pris en compte dans les modèles opérationnels de prévision des surcotes de tempête.

\section{Références bibliographiques}

ABADIE S., BUTEL R., DUPUIS H., BRIÈRE C., (2005). Paramètres statistiques de la houle directionnelle au large de la côte sud aquitaine. Comptes Rendus Geoscience, Vol. 337(8), pp 769-776. http://dx.doi.org/10.1016/j.crte.2005.03.012

APOTSOS A., RAUBENHEIMER B., ELGAR S., GUZA R.T. (2008). Testing and calibrating parametric wave transformation models on natural beaches. Coastal Engineering, Vol. 55(3), pp 224-235. http://dx.doi.org/10.1016/j.coastaleng.2007.10.002

BATTJES J.A., JANSSEN J.P.F.M. (1978). Energy loss and set-up due to breaking of random waves. Proc. 16th Int. Conf. Coastal Engineering, ASCE, pp 569-587.

BERTIN X., BRUNEAU N., BREILH J.-F., FORTUNATO A.B., KARPYTCHEV M., (2012). Importance of wave age and resonance in storm surges: The case Xynthia, Bay of Biscay. Ocean Modelling, Vol. 42, pp 16-30. http://dx.doi.org/10.1016/.ocemod.2011.11.001

BIDLOT J.-R., JANSSEN P., ABDALLA S., (2007). A revised formulation of ocean wave dissipation and its model impact. ECMWF Tech. Memo. 509. ECMWF, Reading, United Kingdom, 27 p. Available online at: http://www.ecmwf.int/publications/

DODET G., BERTIN X., TABORDA R., (2010). Wave climate variability in the NorthEast Atlantic Ocean over the last six decades. Ocean Modelling, Vol. 31(3-4), pp 120131. http://dx.doi.org/10.1016/j.ocemod.2009.10.010

DODET G., BERTIN X., BRUNEAU N., FORTUNATO A.B., NAHON A., ROLAND A., (2013). Wave-current interactions in a wave-dominated tidal inlet. Journal of Geophysical Research: Oceans, Vol. 118(3), pp 1587-1605. http://dx.doi.org/10.1002/jgrc.20146 IRISH J.L., RESIO D.T. (2010). A hydrodynamics-based surge scale for hurricanes. Ocean Engineering, Vol. 37(1), pp 69-81. http://dx.doi.org/10.1016/j.oceaneng.2009.07.012 


\section{XIII ${ }^{\text {èmes }}$ Journées Nationales Génie Côtier - Génie Civil \\ Dunkerque, 2-4 juillet 2014}

KANTHA L., (2013). Classification of hurricanes: Lessons from Katrina, Ike, Irene, Isaac and Sandy. Ocean Engineering, Vol. 70, pp 124-128. http://dx.doi.org/10.1016/j.oceaneng.2013.06.007

LONGUET-HIGGINS M.S., STEWART R.W. (1964). Radiation stresses in water waves; a physical discussion, with applications. Deep Sea Research and Oceanographic Abstracts, Vol. 11(4), pp 529-562. http://dx.doi.org/10.1016/0011-7471(64)90001-4

NICOLLE A. (2006). Modélisation des marées et des surcotes dans les Pertuis charentais. Thèse de doctorat, Université de La Rochelle. 307 p.

NICOLLE A., KARPYTCHEV M., BENOIT M., (2009). Amplification of the storm surges in shallow waters of the Pertuis Charentais (Bay of Biscay, France). Ocean Dynamics, Vol 59(6), pp 921-935. http://dx.doi.org/10.1007/s10236-009-0219-0

POND S., PICKARD G.L. (1998). Introductory Dynamical Oceanography. Butterworth-Heinmann.

ROLAND A., CUCCO A., FERRARIN C., HSU T.-W., LIAU J.-M., OU S.-H., UMGIESSER G., ZANKE U. (2009). On the development and verification of a 2-D coupled wave-current model on unstructured meshes. Journal of Marine Systems, Vol. 78, pp 244-254. http://dx.doi.org/10.1016/i.jmarsys.2009.01.026

SAHA S., MOORTHI S., PAN H.-L., WU X., WANG J., NADIGA S., GOLDBERG M. et al., (2010). The NCEP Climate Forecast System Reanalysis Bulletin of the American Meteorological Society, Vol. 91(8), pp 1015-1057. http://dx.doi.org/10.1175/2010BAMS3001.1

TORRES-FREYERMUTH A., MARIÑO-TAPIA I., CORONADO C., SALLES P., MEDELLÍN G., PEDROZO-ACUÑA A., SILVA R., CANDELA J., IGLESIASPRIETO R. (2012). Wave-induced extreme water levels in the Puerto Morelos fringing reef lagoon. Natural Hazards and Earth System Science, Vol. 12(12), pp 3765-3773. http://dx.doi.org/10.5194/nhess-12-3765-2012

ZHANG Y.J., BAPTISTA A.M., (2008). SELFE : A Semi-Implicit Eulerian Lagrangian Finite-Element Model For Cross-Scale Ocean Circulation. Ocean Modelling, Vol. 21, pp 71-96. http://dx.doi.org/10.1016/j.ocemod.2007.11.005

ZHANG Y.J., WITTER R.C., PRIEST G.R., (2011). Tsunami-tide interaction in 1964 Prince William Sound tsunami. Ocean Modelling, Vol. 40(3-4), pp 246-259. http://dx.doi.org/10.1016/j.ocemod.2011.09.005 
Thème 7 - Risques côtiers 